\title{
Quantum ordering for quantum geodesic functions of orbifold Riemann surfaces
}

\author{
Leonid O. Chekhov ${ }^{\mathrm{ab}}$ and Marta Mazzocco ${ }^{\mathrm{c}}$
}

September 16, 2013

\begin{abstract}
We determine the explicit quantum ordering for a special class of quantum geodesic functions corresponding to geodesics joining exactly two orbifold points or holes on a non-compact Riemann surface. We discuss some special cases in which these quantum geodesic functions form subalgebras of some abstract algebras defined by the reflection equation and we extend our results to the quantisation of matrix elements of the Fuchsian group associated to the Riemann surface in Poincaré uniformization. In particular we explore an interesting relation between the deformed $U_{q}\left(\mathfrak{s l}_{2}\right)$ and the Zhedanov algebra $A W(3)$.
\end{abstract}

For the 75th birthday of Sergei Petrovich Novikov

\section{Introduction}

Convenient Darboux coordinates for Riemann surfaces with holes were identified in [4] with the shear coordinates for an ideal triangle decomposition obtained in [14 by generalising the results obtained for punctured Riemann surfaces in [34].

These coordinates were quantized in [4] and, in a parametrization related to Liouville-type models, in [22]. In all cases, the quantum mapping-class group (MCG) transformations (or, the quantum flip morphsms) that satisfy the quantum pentagon identity involve the quantum dilogarithm function [13. Almost simultaneously, the corresponding Darboux coordinates were identified with the $Y$-type cluster variables [18, [19], and the cluster systems for bordered Riemann surfaces were considered in [17]. The above construction was generalized further to the case of Riemann surfaces with holes and with orbifold points for the case of $\mathbb{Z}_{2}$ and $\mathbb{Z}_{3}$ orbifold points in [2], 3] and for the case or orbifold points of any integer order in [9].

The principal object of investigation for 2D Riemann surfaces are geodesic functions: they are invariant under the MCG action thus constituting the set of observables; in the classical case, the set of lengths of these functions can be identified with the spectrum of a Riemann surface, whereas a quantum Riemann surface is characterized by an algebra of quantum geodesic functions. A simple and explicit construction of the corresponding classical geodesic functions in terms of Darboux coordinates of decorated Teichmüller spaces for Riemann surfaces with holes was proposed in [5]: it was shown

\footnotetext{
${ }^{a}$ Steklov Mathematical Institute and Laboratoire Poncelet, Moscow, Russia. email: chekhov@mi.ras.ru.

${ }^{\mathrm{b}}$ Mathematics Department, Loughborough University, UK. email: l.chekhov@lboro.ac.uk.

${ }^{\mathrm{c}}$ Mathematics Department, Loughborough University, Leicestershire, UK. email: m.mazzocco@lboro.ac.uk.
} 
there that all geodesic functions are Laurent polynomials of exponentiated coordinates with positive integer coefficients; the same remains true for Riemann surfaces with $\mathbb{Z}_{2}$ and $\mathbb{Z}_{3}$ orbifold points whereas the integrity condition breaks in general in the case of orbifold points of arbitrary order [9].

The problem of quantum ordering of a product of noncommuting operators appeared almost simultaneously with the rise of quantum mechanics. In the context of quantum geodesic functions it was first mentioned in [5] where the crucial conditions of mapping-class-group (MCG) invariance and satisfaction of the quantum skein relations were formulated. The compatibility of these two conditions was implicitly proved by Kashaev [23] who constructed unitary operators of quantum Dehn twists whose action on operators of quantum geodesic functions obviously preserves their quantum algebra. It remained however the problem of formulating a recipe for obtaining a quantum operator in an explicit form, likewise the Kulish, Sklyanin, and Nazarov recipe (see [25], [30]) for constructing Yangian central elements extended to the case of reflection equation by Molev, Ragoucy, and Sorba (the quantum ordering for twisted Yangians was constructed in [28] for the $O(n)$ case and in [29] for the $S p(2 n)$ case).

In the present paper, we revise the classical and quantum MCG transformations for Riemann surfaces with holes and orbifold points and, using the results of [8], construct the proper quantum ordering for a special class of geodesic functions corresponding to geodesics going around exactly two orbifold points/holes. We use the same quantum ordering for quantizing matrix elements of the Fuchsian group associated to the Riemann surface. In the case of monodromy matrices, Korotkin and Samtleben in [24] proposed an $r$-matrix structure of the Fock-Rosly type [16] which did not however satisfy Jacobi relations on monodromy matrices themselves but became consistent on the level of adjoint invariant elements. The quantum algebra of entries of the matrices considered in this paper is free of this discrepansy and it is a well-defined quantum algebra. We were able to construct these algebras for matrices in two important cases: in the case of $A_{n}$-algebra related to Schlesinger systems [10] and in the case of the algebra related to the Painlevé VI equation [7]. In the former case, for each fixed geodesic, the matrix entries of the corresponding element in the Fuchsian group satisfy the quantum universal enveloping algebra $U_{q}\left(\mathfrak{s l}_{2}\right)$ relations, and matrix entries coming from different matrices satisfy quantum commutation relations which obey $r$-matrix type relations, thus endowing $U_{q}\left(\mathfrak{s l}_{2}\right) \times \ldots \times U_{q}\left(\mathfrak{s l}_{2}\right)$ with a well defined quantum algebra structure. We also prove that this structure is preserved by the quantum braid group action.

In the case of the algebra related to the Painlevé VI equation, for each fixed geodesic, the matrix entries of the corresponding element in the Fuchsian group satisfy a deformed version of the quantum universal enveloping algebra $U_{q}\left(\mathfrak{s l}_{2}\right)$ relations. This result is quite interesting as it sheds light on the relation between the quantum universal enveloping algebra $U_{q}\left(\mathfrak{s l}_{2}\right)$ and the Zhedanov algebra $A W(3)$ already explored in [38, 35. Indeed, in a recent paper by M.M. [26], it was shown that there is a natural quantisation of the monodromy group associated to the sixth Painlevé equation which leads to the Cherednik algebra of type $\check{C}_{1} C_{1}$. When restricted to the spherical sub-algebra the same quantization corresponds to the isomorphism between quantum geodesic functions on a Riemann sphere with four holes and Zhedanov algebra $A W(3)$ [7, 21].

We leave the case of $D_{n}$-algebras related to the reflection equation with the spectral parameter to subsequent publications.

The structure of the paper is as follows. Section 2 contains a brief review of the fat-graph description of Teichmüller spaces of Riemann surfaces with holes and orbifold points. In Sec. 3, we quantize the MCG transformations for orbifold Riemann surfaces proposed in [9] thus introducing a new class of quantum MCG transformations. In Sec. 4.1, we prove that the corresponding transformations are homogeneous for matrix products of quantum operators. This enables us to prove in Sec. 4.2 the invariance w.r.t. the quantum MCG transformations for the quantum geodesic functions corresponding to geodesics that are homeomorphic to curves separating two orbifold points/holes from the rest of a 
Riemann surface. This class of geodesic functions is of particular interest: for example, it was shown in [6] that such geodesic functions can be identified with elements of particular Poisson leaves of twisted Yangians (see [27] and references therein). Then, in Sec. 5] we construct quantum matrices on rooted fat graphs that are MCG-invariant w.r.t. all transformations that leave the root edge intact. The quantum algebra for the $A_{n}$ case is constructed in Sec. 5.1 and we represent it in the $R$-matrix form in Sec. 5.2 thus constructing the consistent quantum algebra for monodromy matrices. We present the action of the quantum braid group transformations on the level of monodromy matrices in Sec. 5.3, In Sec. 5.4, we find the quantum algebra for monodromy matrices of the Painlevé VI equation.

\section{Combinatorial description of $\mathfrak{T}_{g, s, r}^{H}$}

\subsection{Fat graph description for Riemann surfaces with holes and $\mathbb{Z}_{p}$ orbifold points}

Definition 1. We call a fat graph (a graph with the prescribed cyclic ordering of edges entering each vertex) $\Gamma_{g, s, r}$ a spine of the Riemann surface $\Sigma_{g, s, r}$ with $g$ handles, $s>0$ holes, and $r$ orbifold points of the corresponding orders $p_{i}, i=1, \ldots, r$, if

(a) this graph can be embedded without self-intersections in $\Sigma_{g, s, r}$;

(b) all vertices of $\Gamma_{g, s, r}$ are three-valent except exactly $r$ one-valent vertices (endpoints of "pending" edges), which are placed at the corresponding orbifold points;

(c) upon cutting along all edges of $\Gamma_{g, s, r}$ the Riemann surface $\Sigma_{g, s, r}$ splits into $s$ polygons each containing exactly one hole and being simply connected upon contracting this hole.

Because every pending edge "protrudes" towards the interior of some face of the graph and every face contains exactly one hole, the above fat graph determines a natural partition of the set of orbifold points into nonintersecting (maybe empty) subsets $\delta_{k}, k=1, \ldots, s$ of orbifold points incident to the corresponding face (boundary component, or hole). Edges of the above graph are labeled by distinct integers $\alpha=1,2, \ldots, 6 g-6+3 s+2 r$, and we set a real number $Z_{\alpha}$ into correspondence to the $\alpha$ th edge.

The first homotopy groups $\pi_{1}\left(\Sigma_{g, s, r}\right)$ and $\pi_{1}\left(\Gamma_{g, s, r}\right)$ coincide because each closed path in $\Sigma_{g, s, r}$ can be homotopically transformed to a closed path in $\Gamma_{g, s, r}$ (taking into account paths that go around orbifold points) in a unique way. The standard statement in hyperbolic geometry is that conjugacy classes of elements of a Fuchsian group $\Delta_{g, s, r}$ are in the 1-1 correspondence with homotopy classes of closed paths in the Riemann surface $\Sigma_{g, s, r}=\mathbb{H}_{+}^{2} / \Delta_{g, s, r}$ and that the "actual" length $\ell_{\gamma}$ of a hyperbolic element $\gamma \in \Delta_{g, s, r}$ coincides with the minimum length of curves from the corresponding homotopy class; it is then the length of a unique closed geodesic line belonging to this class.

The real numbers $Z_{\alpha}$ in Definition 1 are the $h$-lengths (logarithms of cross-ratios) [34]: they are called the (Thurston) shear coordinates [36, [1] in the case of punctured Riemann surface. Below we identify these shear coordinates with coordinates of the decorated Teichmüller space $\mathfrak{T}_{g, s, r}^{H}$.

\subsection{The Fuchsian group $\Delta_{g, s, r}$ and geodesic functions}

We now describe combinatorially the conjugacy classes of the Fuchsian group $\Delta_{g, s, r}$. Every time the path homeomorphic to a (closed) geodesic $\gamma$ passes along the edge with the label $\alpha$ we insert [14] the so-called edge matrix, i.e. the matrix of Möbius transformation:

$$
X_{Z_{\alpha}}=\left(\begin{array}{cc}
0 & -\mathrm{e}^{Z_{\alpha} / 2} \\
\mathrm{e}^{-Z_{\alpha} / 2} & 0
\end{array}\right)
$$




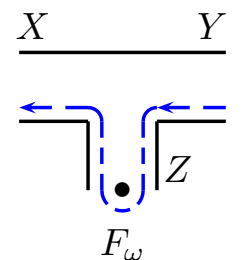

(a)

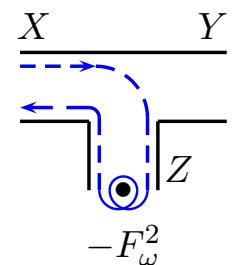

(b)

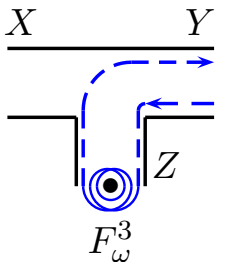

$(\mathrm{c})$

Figure 1: Part of a graph with a pending edge. Its endpoint with the orbifold point is directed toward the interior of the boundary component this point is associated with. The variable $Z$ corresponds to the respective pending edge. We present three typical examples of geodesics undergoing single (a), double (b), and triple (c) rotations at the $\mathbb{Z}_{p}$ orbifold point.

into the corresponding string of matrices. We also have the "right" and "left" turn matrices to be set in proper places when a path makes corresponding turns at three-valent vertices,

$$
R=\left(\begin{array}{cc}
1 & 1 \\
-1 & 0
\end{array}\right), \quad L=R^{2}=\left(\begin{array}{cc}
0 & 1 \\
-1 & -1
\end{array}\right)
$$

When orbifold points are present, the Fuchsian group contains besides hyperbolic elements also elliptic elements corresponding to rotations about these orbifold points. The corresponding generators $\widetilde{F}_{i}, i=1, \ldots, r$, of the rotations through $2 \pi / p_{i}$ are conjugates of the matrices

$$
\widetilde{F}_{i}=U_{i} F_{\omega_{i}} U_{i}^{-1}, \quad F_{\omega}:=\left(\begin{array}{cc}
0 & 1 \\
-1 & -w
\end{array}\right), \quad w=2 \cos \pi / p \text { for some } p \geq 2 .
$$

New elements of the Fuchsian group correspond to rotations of geodesics when going around orbifold points indicated by dot-vertices; for a $\mathbb{Z}_{p}$ orbifold point we then insert the above matrix $F_{\omega}$ into the corresponding string of $2 \times 2$-matrices (when we go around the orbifold point counterclockwise as in Fig. 1(a)). When going around an orbifold point $k$ times we insert the matrix $(-1)^{k+1} F_{\omega}^{k}$ into the product of $2 \times 2$-matrices. For example, parts of geodesic functions in the three cases in Fig. 1 read

$$
\begin{aligned}
& \text { (a) } \ldots X_{X} L X_{Z} F_{\omega} X_{Z} L X_{Y} \ldots \\
& \text { (b) } \ldots X_{X} L X_{Z}\left(-F_{\omega}^{2}\right) X_{Z} R X_{X} \ldots \\
& \text { (c) } \ldots X_{Y} R X_{Z}\left(F_{\omega}^{3}\right) X_{Z} L X_{Y} \ldots
\end{aligned}
$$

Note that for $\omega_{p}=2 \cos \pi / p, F_{\omega_{p}}^{p}=(-1)^{p-1} \mathbb{E}$, so going around the $\mathbb{Z}_{p}$ orbifold point $p$ times merely corresponds to avoiding this orbifold point due to the simple equality (note that $X_{S}^{2}=-\mathbb{E}$ and $\left.L^{2}=-R\right)$

$$
X_{X} L X_{Z}(-1)^{p-1} F_{\omega_{p}}^{p} X_{Z} L X_{Y}=X_{X} L X_{Z}^{2} L X_{Y}=-X_{X} L^{2} X_{Y}=X_{X} R X_{Y}
$$

(For the $\mathbb{Z}_{2}$ orbifold points this pattern was first proposed by Fock and Goncharov [15]; the graph morphisms were described in [3].)

An element of a Fuchsian group has then the typical structure

$$
P_{\gamma}=L X_{Y_{n}} R X_{Y_{n-1}} \cdots R X_{Y_{2}} L X_{Z_{1}}(-1)^{k+1} F_{\omega_{i}}^{k} X_{Z_{1}} R X_{Y_{1}},
$$

where $Y_{i}$ are variables of "internal" edges and $Z_{j}$ are those of pending edges. In the corresponding geodesic function

$$
G_{\gamma} \equiv \operatorname{tr} P_{\gamma}=2 \cosh \left(\ell_{\gamma} / 2\right)
$$

$\ell_{\gamma}$ is the actual length of the closed geodesic on the Riemann surface. 
Remark 2. Note that the combinations

$$
\begin{aligned}
R X_{Y} & =\left(\begin{array}{cc}
e^{-Y / 2} & -e^{Y / 2} \\
0 & e^{Y / 2}
\end{array}\right), \quad \text { and } L X_{Y}=\left(\begin{array}{cc}
e^{-Y / 2} & 0 \\
-e^{-Y / 2} & e^{Y / 2}
\end{array}\right), \\
R X_{Z} F_{\omega} X_{Z} & =\left(\begin{array}{cc}
e^{-Z}+\omega & -e^{Z} \\
-\omega & e^{Z}
\end{array}\right) \text { and } L X_{Z} F_{\omega} X_{Z}=\left(\begin{array}{cc}
e^{-Z} & 0 \\
-e^{-Z}-\omega & e^{Z}
\end{array}\right),
\end{aligned}
$$

as well as products of any number of these matrices have the sign structure $\left(\begin{array}{c}+ \\ - \\ -\end{array}\right)$, so the trace of any of $P_{\gamma}$ with first powers of $F_{\omega}$ is a sum of exponentials with positive integer coefficients; this sum always include the terms

$$
e^{Y_{1} / 2+\cdots+Y_{n} / 2+Z_{1}+\cdots+Z_{s}} \quad \text { and } e^{-Y_{1} / 2-\cdots-Y_{n} / 2-Z_{1}-\cdots-Z_{s}}
$$

being therefore always greater or equal two thus describing a hyperbolic or parabolic element; the latter is possible only if $Y_{1}+\cdots+Y_{n}+Z_{1}+\cdots+Z_{s}=0$ and only if all the turn matrices in (5) are left-turn ones (for our choice of $F_{\omega}$; when going in the opposite direction we must insert $F_{\omega}^{-1}$, and all the turn matrices must be right-turn ones), which corresponds to a path going along the boundary of a face; all such paths are homeomorphic to the hole boundaries, and the condition that the sum of $Y_{i}$ and $Z_{j}$ equals zero along this path indicates the degeneration of a hole into a puncture. Fot the complete proof of hyperbolicity of all elements not homeomorphic to going several times around a single orbifold point, see [9].

The group generated by elements (3) together with translations along $A$ - and $B$-cycles and around holes not necessarily produces a regular (metrizable) surface because its action is not necessarily discrete. The necessary and sufficient conditions for producing a regular surface in terms of graphs were formulated as a theorem in [9]. (We call a Riemann surface regular if it is locally a smooth constant-curvature surface everywhere except exactly $r$ orbifold points.) Essentially, it was proved that we obtain a regular Riemann surface for any choice of real numbers $Y_{i}$ and $Z_{\alpha}$ from Definition 1 and vice versa, for any regular Riemann surface $\Sigma_{g, s, r}=\mathbb{H}_{+}^{2} / \Delta_{g, s, r}$ and for any fat graph that is a spine of this surface we find a set of real numbers $Y_{i}$ and $Z_{\alpha}$ that are the shear coordinates for an ideal triangle decomposition of the above surface. This set is not unique; however the equivalent sets are related by discrete modular group action, and we can therefore identify the $(6 g-6+3 s+2 r)$ tuple of real coordinates $\left\{Y_{i}, Z_{\alpha}\right\}$ with the coordinates of the decorated Teichmüller space $\mathfrak{T}_{g, s, r}^{H}$ (the decoration assigns positive or negative signs to every hole with nonzero perimeter). The lengths of geodesics on $\Sigma_{g, s, r}$ are given by traces of products (5) corresponding to paths in the corresponding spine.

We see that every spine $\Gamma_{g, s, r}$ provides a parameterization of the (decorated) Teichmüller space $\mathfrak{T}_{g, s, r}^{H}$. Transitions between different parameterizations are formulated in terms of flips (mutations) of edges: any two spines from the given topological class are related by a finite sequence of flips. We therefore identify flips of edges with the action of the MCG to be constructed in the next section.

\section{Quantum mapping class group transformations for surfaces with orbifold points}

\subsection{Poisson structure}

One of the most attractive properties of the graph description is a very simple Poisson algebra on the set of parameters $Z_{\alpha}, \alpha=1, \ldots, 6 g-6+3 s+2 r$ (we do not distinguish here the parameters of internal 
and pending edges).

Theorem 1. In the coordinates $Z_{\alpha}$ on any fixed spine corresponding to a surface with or without orbifold points, the Weil-Petersson bracket $B_{W P}$ reads

$$
\{f(\mathbf{Z}), g(\mathbf{Z})\}=\sum_{\begin{array}{c}
3 \text {-valent } \\
\text { vertices } \alpha=1
\end{array}}^{4 g+2 s+|\delta|-4} \sum_{i=1}^{3 \bmod 3}\left(\frac{\partial f}{\partial Z_{\alpha_{i}}} \frac{\partial g}{\partial Z_{\alpha_{i+1}}}-\frac{\partial g}{\partial Z_{\alpha_{i}}} \frac{\partial f}{\partial Z_{\alpha_{i+1}}}\right),
$$

where the sum ranges all the three-valent vertices of a graph and $\alpha_{i}$ are the labels of the cyclically (clockwise) ordered $\left(\alpha_{4} \equiv \alpha_{1}\right)$ edges incident to the vertex with the label $\alpha$. The formula (7) is insensitive to whether these edges are internal or pending edges. This bracket gives rise to the Goldman bracket on the space of geodesic length functions [20].

The center of the Poisson algebra (7) is generated by elements of the form $\sum Z_{\alpha}$, where the sum ranges all edges of $\Gamma_{g, s, r}$ belonging to the same boundary component taken with multiplicities. This means, in particular, that each pending edge, irrespectively on the type of orbifold point it corresponds to, contributes twice to such sums. The dimension of this center is obviously $s$.

Note that for the path homeomorphic to the hole boundary, for any number of insertions of matrices $F_{p_{i}}$ with any $p_{i}$, we have

$$
\begin{aligned}
& \operatorname{tr}\left[L X_{Z_{1}} L X_{Z_{2}} \cdots L X_{Z_{k}} F_{\omega} X_{Z_{k}} L \cdots L X_{Z_{n-1}} L X_{Z_{n}}\right] \\
& \quad=2 \cosh \left[Z_{1} / 2+Z_{2} / 2+\ldots+Z_{k}+\ldots+Z_{n-1} / 2+Z_{n} / 2\right]
\end{aligned}
$$

\subsection{Flip morphisms of fat graphs}

We now describe all mapping class group transformations that enable us to change numbers $\left|\delta_{k}\right|$ of orbifold points associated with the $k$ th hole, change the cyclic ordering inside any of the sets $\delta_{k}$, flip any inner edge of the graph and, eventually, change the decoration, i.e., orientation of the geodesic spiraling to the hole perimeter (in the case where we have more than one hole). We can therefore establish a morphism between any two of the graphs belonging to the same class $\Gamma_{g, s, r}$ with the same (unordered) sets of orbifold point orders $\left\{p_{i}\right\}_{i=1}^{r}$.

\subsubsection{Flipping inner edges}

Given a spine $\Gamma$ of $\Sigma$ and assuming that the internal edge $\alpha$ has distinct endpoints, we may produce another spine $\Gamma_{\alpha}$ of $\Sigma$ by contracting and expanding edge $\alpha$ of $\Gamma$, the edge labeled $Z$ in Figure 2 , We say that $\Gamma_{\alpha}$ arises from $\Gamma$ by a Whitehead move (or flip) along the edge $\alpha$. A labeling of edges of the spine $\Gamma$ implies a natural labeling of edges of the spine $\Gamma_{\alpha}$; we then obtain a morphism between the spines $\Gamma$ and $\Gamma_{\alpha}$.

It was shown in [4] that setting $\phi(Z)=\log \left(1+e^{Z}\right)$ and adopting the notation of Fig. 2 for shear coordinates of nearby edges, the effect of a flip is

$$
\begin{aligned}
W_{Z}:(A, B, C, D, Z) & \rightarrow(A+\phi(Z), B-\phi(-Z), C+\phi(Z), D-\phi(-Z),-Z) \\
& :=(\tilde{A}, \tilde{B}, \tilde{C}, \tilde{D}, \tilde{Z}) .
\end{aligned}
$$

In various cases where the edges are not distinct, we have: if $A=C$, then $\tilde{A}=A+2 \phi(Z)$; if $B=D$, then $\tilde{B}=B-2 \phi(-Z)$; if $A=B($ or $C=D)$, then $\tilde{A}=A+Z($ or $\tilde{C}=C+Z)$; if $A=D($ or $B=C)$, then $\tilde{A}=A+Z$ (or $\tilde{B}=B+Z$ ). Any subset of edges $A, B, C$, and $D$ can be pending edges of the graph.

We have the lemma establishing the properties of invariance w.r.t. the flip morphisms [5]. 


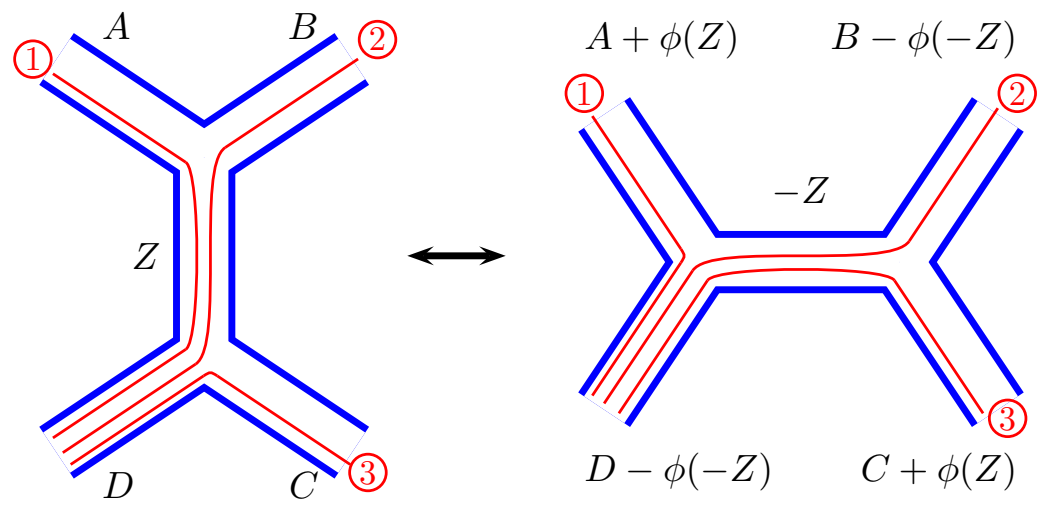

Figure 2: Flip on the shear coordinates $Z_{\alpha}$. The outer edges can be pending, but the edge undergoing the flip must be an internal edge with distinct endpoints. We also indicate the correspondences between geodesic paths under the flip.

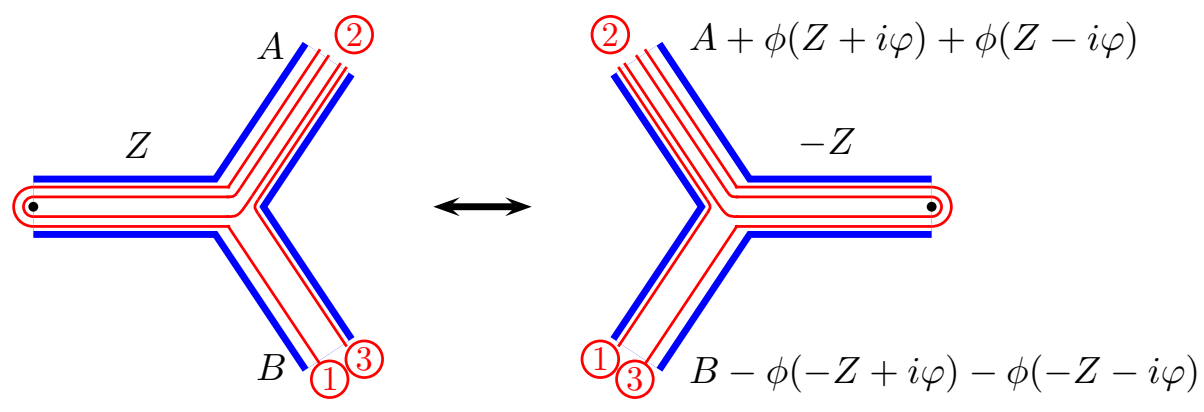

Figure 3: The transformation of dual variables ( $h$-lengths) when flipping a pending edge; $w=2 \cos (\pi / p)$ and $\varphi=\pi / p$. We indicate the orbifold point by the bullet and demonstrate the changing of geodesic functions upon flipping the edge.

Lemma 2. Transformation (8) preserves the traces of products over paths (6) (the geodesic functions) and transformation (8) simultaneously preserves Poisson structure (7) on the shear coordinates.

The proof of this lemma is based on the following useful matrix equalities, which are valid without taking the trace (they correspond to three geodesic cases in Fig. 2):

$$
\begin{aligned}
X_{D} R X_{Z} R X_{A} & =X_{\tilde{A}} R X_{\tilde{D}}, \\
X_{D} R X_{Z} L X_{B} & =X_{\tilde{D}} L X_{\tilde{Z}} R X_{\tilde{B}}, \\
X_{C} L X_{D} & =X_{\tilde{C}} L X_{\tilde{Z}} L X_{\tilde{D}}
\end{aligned}
$$

\subsubsection{Flipping pending edges}

Lemma 3. The transformation in Fig. 3

$$
\{\tilde{A}, \tilde{B}, \tilde{Z}\}:=\{A+\phi(Z+i \varphi)+\phi(Z-i \varphi), B-\phi(-Z+i \varphi)-\phi(-Z-i \varphi),-Z\},
$$


where $\phi(x)=\log \left(1+e^{x}\right)$ and $w=e^{i \varphi}+e^{-i \varphi}$, is the morphism of the space $\mathfrak{T}_{g, s, r}^{H}$. These morphisms preserve both Poisson structures (7) and the geodesic functions. In Fig. 3 any (or both) of variables $A$ and $B$ can be variables of pending edges (the transformation formula is insensitive to it).

In this case, again, verifying the preservation of Poisson relations (7) is simple, whereas for traces over paths we have three different cases, and in each of these cases we again obtain $2 \times 2$-matrix equalities to be verified directly

$$
\Omega=\left(\begin{array}{cc}
a & c \\
-c & a-w c
\end{array}\right), \quad \forall a, c \in \mathbb{C},
$$

denote any matrix commuting with $F_{p}$ (in particular, $F_{p}^{k}, k \in \mathbb{Z}$ ). We then have the following matrix equalities:

$$
\begin{aligned}
X_{A} L X_{Z}\left(F_{p} \Omega\right) X_{Z} L X_{B} & =X_{\tilde{A}} R X_{\tilde{Z}}(-\Omega) X_{\tilde{Z}} R X_{\tilde{B}} \\
X_{A} L X_{Z} \Omega X_{Z} R X_{A} & =X_{\tilde{A}} R X_{\tilde{Z}}(-\Omega) X_{\tilde{Z}} L X_{\tilde{A}} \\
X_{B} R X_{Z} \Omega X_{Z} L X_{B} & =X_{\tilde{B}} L X_{\tilde{Z}}(-\Omega) X_{\tilde{Z}} R X_{\tilde{B}}
\end{aligned}
$$

Using flip morphisms in Fig. 3 and in formula (8), we establish a morphism between any two algebras corresponding to surfaces of the same genus, same number of boundary components, and same numbers of $\mathbb{Z}_{p}$-orbifold points of each sort $p$; the distribution of latter into the boundary components as well as the cyclic ordering inside each of the boundary component can be arbitrary.

It is a standard tool that if, after a series of morphisms, we come to a graph of the same combinatorial type as the initial one (disregarding labeling of edges but distinguishing between different orbifold types of pending vertices), we associate a mapping class group operation to this morphism therefore passing from the groupoid of morphisms to the group of modular transformations.

\subsubsection{Changing the decoration (spiraling direction)}

The last class of mapping class group transformations pertains to changing the "decoration," i.e., the sign of the hole perimeter:

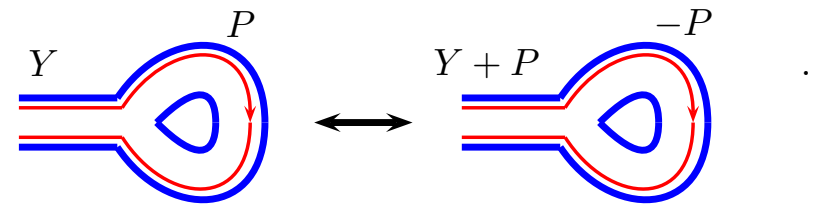

That this transformation preserves the Poisson brackets is obvious because the variable $P$ Poisson commutes with all other variables, whereas the preservation of geodesic functions follows from two matrix equalities:

$$
\begin{aligned}
& X_{Y} L X_{P} L X_{Y}=X_{Y+P} L X_{-P} L X_{Y+P} \\
& X_{Y} R X_{P} R X_{Y}=X_{Y+P} R X_{-P} R X_{Y+P}
\end{aligned}
$$

We can therefore extend the mapping class group of $\mathfrak{T}_{g, s, r}^{H}$ by adding symmetries between sheets of the $2^{s}$-ramified covering of the "genuine" (nondecorated) Teichmüller space $\mathfrak{T}_{g, s, r}$.

We can summarize as follows.

Theorem 4. The whole mapping class group of $\Sigma_{g, s, r}$ is generated by morphisms described by Lemmas 2 and 3 and formula (16).

\footnotetext{
${ }^{\mathrm{a}}$ In [9], the powers of the matrix $F_{p}$ were incorrect in the corresponding formula.
} 


\subsection{Quantum MCG transformations}

Recall that a quantization of a Poisson manifold equivariant w.r.t. a discrete group action is a family of *-algebras $\mathcal{A}^{\hbar}$ depending smoothly on a positive real parameter $\hbar$, acting on $G$ by outer automorphisms and having the following relation to the Poisson manifold.

1. For $\hbar=0$, the algebra is isomorphic as a $G$-module to the $*$-algebra of complex-valued function on the Poisson manifold.

2. The Poisson bracket on $\mathcal{A}^{0}$ given by $\left\{a_{1}, a_{2}\right\}=\lim _{\hbar \rightarrow 0} \frac{\left[a_{1}, a_{2}\right]}{\hbar}$ coincides with the one generated by the Poisson structure of the manifold.

We now quantize a Teichmüller space $\mathfrak{T}_{g, s, r}^{H}$ equivariantly w.r.t. the mapping class group action.

Let $\mathfrak{T}^{\hbar}\left(\Gamma_{g, s, r}\right)$ be a $*$-algebra generated by the generator $Z_{\alpha}^{\hbar}$ (one generator per one unoriented edge $\alpha$ ) and relations

$$
\left[Z_{\alpha}^{\hbar}, Z_{\beta}^{\hbar}\right]=2 \pi i \hbar\left\{Z_{\alpha}, Z_{\beta}\right\}
$$

with the $*$-structure

$$
\left(Z_{\alpha}^{\hbar}\right)^{*}=Z_{\alpha}^{\hbar}
$$

Here $z_{\alpha}$ and $\{\cdot, \cdot\}$ stand for the respective coordinate functions on the classical Teichmüller space and the Weil-Petersson Poisson bracket on it. Note that according to formula (7), the right-hand side of (17) is merely a constant which may take only five values $0, \pm 2 \pi i \hbar, \pm 4 \pi i \hbar$.

For the notation simplicity in what follows we omit the superscript $\hbar$ for the quantum operators; the classical or quantum nature of the object will be always clear from the context.

\subsubsection{Quantum flip morphisms for inner edges}

It was proved in [4] that the quantum flip morphisms

$$
\begin{aligned}
\{A, B, C, D, Z\} & \rightarrow\left\{A+\phi^{\hbar}(Z), B-\phi^{\hbar}(-Z), C+\phi^{\hbar}(Z), D-\phi^{\hbar}(-Z),-Z\right\} \\
& :=\{\tilde{A}, \tilde{B}, \tilde{C}, \tilde{D}, \tilde{Z}\},
\end{aligned}
$$

where $A, B, C, D$, and $Z$ are as in Fig. 2 and $\phi^{\hbar}(x)$ is the real function of one real variable,

$$
\phi^{\hbar}(z)=-\frac{\pi \hbar}{2} \int_{\Omega} \frac{e^{-i p z}}{\sinh (\pi p) \sinh (\pi \hbar p)} d p
$$

(the contour $\Omega$ goes along the real axis bypassing the singularity at the origin from above) satisfy the standard two-, four-, and five-term relations. The quantum dilogarithm function $\phi^{\hbar}(z)$ was introduced in this context by Faddeev in [13] and used in [12] for constructing quantum MCG transformations for the Liouville model.

As in the classical case, the center of the algebra is generated by the sums $Z_{f}^{\hbar}:=\sum_{\alpha \in f} Z_{\alpha}^{\hbar}$ ranging all edges $\alpha$ surrounding a given face $f$.

Important properties of the quantum flip morphisms are

- In the limit $\hbar \rightarrow 0$, morphism (19) coincides with the classical morphism, that is,

$$
\lim _{\hbar \rightarrow 0} \phi^{\hbar}(z)=\log \left(e^{z}+1\right)
$$

- The morphism of the commutation relations is ensured by the relation

$$
\phi^{\hbar}(z)-\phi^{\hbar}(-z)=z .
$$


- The Hermiticity of the transformed operators is ensured by the conjugation condition

$$
\overline{\phi^{\hbar}(z)}=\phi^{\hbar}(\bar{z})
$$

- Eventually, we have the double quasi-periodicity conditions responsible for the satisfaction of the five-term relation:

$$
\begin{gathered}
\phi^{\hbar}(z+i \pi \hbar)-\phi^{\hbar}(z-i \pi \hbar)=\frac{2 \pi i \hbar}{e^{-z}+1}, \\
\phi^{\hbar}(z+i \pi)-\phi^{\hbar}(z-i \pi)=\frac{2 \pi i}{e^{-z / \hbar}+1} .
\end{gathered}
$$

- The function $\phi^{\hbar}(z)$ is meromorphic with poles at the points $\{\pi i(m+n \hbar) \mid m, n \in \mathbf{N}\}$ and $\{-\pi i(m+$ $n \hbar) \mid m, n \in \mathbf{N}\}$.

We also have conditions of the modular double, $\frac{1}{\hbar} \phi^{\hbar}(z)=\phi^{1 / \hbar}(z / \hbar)$ and the first quasi-periodicity condition (21), which are irrelevant when we discuss geodesic functions on which half of the modular double generators act trivially.

Remark 3. Note that exponentiated algebraic elements $U_{i}=e^{ \pm Z_{i}}$, which obey homogeneous commutation relations $q^{n} U_{i} U_{j}=U_{j} U_{i} q^{-n}$ with $\left[X_{i}, X_{j}\right]=2 n i \pi \hbar$ and $q:=e^{i \pi \hbar}$ transform as rational functions: for example,

$$
\begin{aligned}
e^{A+\phi^{\hbar}(Z)} & =\exp \left(\frac{1}{2 \pi i \hbar} \int_{A}^{A+2 i \pi \hbar} \phi^{\hbar}(z) d z\right) e^{A}= \\
& =\exp \left(\frac{1}{2 i \pi \hbar} \int_{-\infty}^{A}\left(\phi^{\hbar}(z+2 i \pi \hbar)-\phi^{\hbar}(z)\right) d z\right) e^{A}= \\
& =\exp \left(\int_{-\infty}^{A} \frac{d z}{e^{-z-\pi i \hbar}+1}\right) e^{A}=\left(1+q e^{Z}\right) e^{A}
\end{aligned}
$$

where we have used the standard formula

$$
e^{A+\Phi(B)}=\exp \left\{\frac{1}{[A, B]} \int_{B}^{B+[A, B]} \Phi(z) d z\right\} e^{A},
$$

which is valid for all $A$ and $B$ such that the commutator $[A, B]$ is a nonzero scalar.

\subsubsection{Quantum flip morphisms for pending edges}

We now present the new formula, which is the quantization of the MCG transformation in Fig. 3.

Lemma 5. The transformation in Fig. 3

$$
\{\tilde{A}, \tilde{B}, \tilde{Z}\}:=\left\{A+\phi^{\hbar}(Z+i \varphi)+\phi^{\hbar}(Z-i \varphi), B-\phi^{\hbar}(-Z+i \varphi)-\phi^{\hbar}(-Z-i \varphi),-Z\right\},
$$

with $\phi^{\hbar}(x)$ from (20) and $w=e^{i \varphi}+e^{-i \varphi}$, is the morphism of the quantum $*$-algebra $\mathfrak{T}_{g, s, r}^{\hbar}$.

Verifying the preservation of the commutation relations is simple, the most difficult part is to verify the preservation of geodesic function operators. We devote a special section to follow to the verification of this condition. 


\section{Operator ordering for quantum geodesics}

\subsection{Transformation properties of quantum geodesic functions}

The quantum analogues of matrix relations (99)-(11) were found in [8]. Here and hereafter, for the rest of the paper, we assume that the ordering of quantum operators in a product is natural, i.e., it is determined by the order of matrix multiplication itself. Then, amazingly enough, all four entries of the corresponding $2 \times 2$-matrices transform uniformly: applying the quantum MCG transformation to curves 1,2, and 3 in Fig. 2, we obtain the respective quantum matrix relations:

$$
\begin{aligned}
X_{D} R X_{Z} R X_{A} & =q^{1 / 4} X_{\tilde{D}} R X_{\tilde{A}} \\
X_{D} R X_{Z} L X_{B} & =X_{\tilde{D}} R X_{\tilde{Z}} L X_{\tilde{B}} \\
X_{D} L X_{C} & =q^{1 / 4} X_{\tilde{D}} L X_{\tilde{Z}} L X_{\tilde{C}}
\end{aligned}
$$

We prove here the third relation; the remaining relations can be verified analogously. In the left-hand side, we obtain, after reducing the matrix elements to the Weyl-ordered form, that

$$
\begin{aligned}
X_{\tilde{D}} L X_{\tilde{Z}} L X_{\tilde{C}} & =\left[\begin{array}{cc}
e^{\tilde{D} / 2-\tilde{C} / 2-\tilde{Z} / 2}+e^{\tilde{D} / 2-\tilde{C} / 2+\tilde{Z} / 2} & -q^{-1 / 2} e^{\tilde{D} / 2+\tilde{C} / 2+\tilde{Z} / 2} \\
-q^{-1 / 2} e^{-\tilde{D} / 2-\tilde{C} / 2-\tilde{Z} / 2} & 0
\end{array}\right] \\
& =\left[\begin{array}{cc}
e^{D / 2-C / 2} & -q^{-1 / 2} e^{D / 2+C / 2} \\
-q^{-1 / 2} e^{-D / 2-C / 2} & 0
\end{array}\right],
\end{aligned}
$$

where we have used the relation $\tilde{D}+\tilde{C}+\tilde{Z}=D+C$ and the chain of equalities

$$
\begin{aligned}
& e^{\tilde{D} / 2-\tilde{C} / 2-\tilde{Z} / 2}+e^{\tilde{D} / 2-\tilde{C} / 2+\tilde{Z} / 2}=e^{Z+D / 2-C / 2-\phi^{\hbar}(Z)}+e^{D / 2-C / 2-\phi^{\hbar}(Z)} \\
& =\exp \left\{\frac{1}{2 \pi i \hbar} \int_{Z}^{Z-2 \pi i \hbar}\left[-\xi+\phi^{\hbar}(\xi)\right] d \xi\right\} e^{D / 2-C / 2}+\exp \left\{\frac{1}{2 \pi i \hbar} \int_{Z}^{Z-2 \pi i \hbar} \phi^{\hbar}(\xi) d \xi\right\} e^{D / 2-C / 2} \\
& =\left[e^{Z-i \pi \hbar}+1\right] \exp \left\{\frac{1}{2 \pi i \hbar} \int_{-\infty}^{Z} \frac{-2 \pi i \hbar d \xi}{e^{-\xi+i \pi \hbar}+1}\right\} e^{D / 2-C / 2} \\
& =\left[e^{Z-i \pi \hbar}+1\right] \frac{1}{e^{Z-i \pi \hbar}+1} e^{D / 2-C / 2}=e^{D / 2-C / 2} .
\end{aligned}
$$

In the right-hand side, we have

$$
X_{D} L X_{C}=\left[\begin{array}{cc}
q^{1 / 4} e^{D / 2-C / 2} & -q^{-1 / 4} e^{C / 2+D / 2} \\
-q^{-1 / 4} e^{-C / 2-D / 2} & 0
\end{array}\right]
$$

and, comparing powers of $q$ standing with each of the four matrix entries, we come to the matrix equality (27).

We now formulate the new lemma concerning quantum versions of MCG transformations (13)-(15).

Lemma 6. We have the following quantum matrix relations:

$$
\begin{aligned}
X_{A} L X_{Z}\left(F_{p} \Omega\right) X_{Z} L X_{B} & =q^{-1} X_{\tilde{A}} R X_{\tilde{Z}}(-\Omega) X_{\tilde{Z}} R X_{\tilde{B}}, \\
X_{A} L X_{Z} \Omega X_{Z} R X_{A} & =X_{\tilde{A}} R X_{\tilde{Z}}(-\Omega) X_{\tilde{Z}} L X_{\tilde{A}}, \\
X_{B} R X_{Z} \Omega X_{Z} L X_{B} & =X_{\tilde{B}} L X_{\tilde{Z}}(-\Omega) X_{\tilde{Z}} R X_{\tilde{B}} .
\end{aligned}
$$

The proof is again a direct calculation. 
Remark 4. A closer look on transformation laws (25)-(27) and (28)-(30) yields a "mnemonic" rule for the powers of $q$ : the "proper" power of $q$ is always one fourth of the difference between the number of matrices $R$ and $L$ in the matrix chain. Although for the general geodesic functions this property presumably does not lead to meaningful answers coming into contradiction with the Hermiticity condition, for a special class of geodesic functions in the next section it enables us to solve the problem of quantum ordering completely.

\subsection{Quantum ordering for special class of geodesic functions}

Quantum matrix MCG relations turn out to be very useful when we consider special geodesic functions that correspond to geodesics going around exactly two orbifold points or holes. Fortunately, many important geodesic functions fall into this class, including those appearing in the description of Poisson leaves of twisted Yangian systems of $A_{n}$ and $D_{n}$ types [6] and those pertaining to the description of Painlevé VI equation [7]. Our aim in this and the next section is to introduce a proper quantum ordering not only for quantum geodesic functions but for more general monodromy matrices of Schlesinger systems (we then obtain geodesic functions as traces of products of these monodromy matrices).

We first take one of these points (the zeroth orbifold point) as a root, let $S:=Z_{0}$ be the variable of the pending edge terminating at this point, and consider $2 \times 2$-monodromy matrices $M_{i}$ corresponding to going around an $i$ th orbifold point. These matrices have the structure

$$
M_{i}=X_{S} R X_{Y_{1}} L \cdots R X_{Y_{p}} L X_{Z_{i}} F_{\omega_{i}} X_{Z_{i}} R X_{Y_{p}} L \cdots R X_{Y_{1}} L X_{S}, i \neq 0, \quad M_{0}=F_{\omega_{0}}
$$

where $Z_{i}$ is the variable of the pending edge terminating at the $i$ th orbifold point and $Y_{1}, \ldots, Y_{p}$ are variables of the intermediate (not necessarily internal) edges. In the general situation we require the path joining the root and the orbifold point neither to be unique (this is true only for fat graphs without loops) nor to have no self-intersections. Whatever is this path, from the transformation properties of quantum geodesic functions we can immediately conclude that any product of quantum matrices of the form (31) is invariant under all the quantum MCG transformations that do not mutate the root edge. For a path without self-intersections, using quantum MCG transformations we can reduce any string of quantum matrices (31) to

$$
M_{i}=X_{\tilde{S}} L X_{\tilde{Z}_{i}} F_{\omega_{i}} X_{\tilde{Z}_{i}} R X_{\tilde{S}}=\left(\begin{array}{cc}
q e^{-\tilde{Z}_{i}}+\omega_{i} & -e^{-\tilde{Z}_{i}+\tilde{S}}-e^{\tilde{Z}_{i}+\tilde{S}}-\omega_{i} e^{\tilde{S}} \\
e^{-\tilde{Z}_{i}-\tilde{S}} & -q^{-1} e^{-\tilde{Z}_{i}}
\end{array}\right), \quad\left[\tilde{Z}_{i}, \tilde{S}\right]=2 \pi i \hbar .
$$

Introducing the notation

$$
a_{i}:=e^{-\tilde{Z}_{i}}, \quad b_{i}=e^{-\tilde{Z}_{i}+\tilde{S}}+e^{\tilde{Z}_{i}+\tilde{S}}+\omega_{i} e^{\tilde{S}}, \quad c_{i}:=e^{-\tilde{Z}_{i}-\tilde{S}},
$$

where $a_{i}, b_{i}$, and $c_{i}$ are Hermitian operators, we eventually construct from the entries of the monodromy matrix (32) (or (31)) the quantum geodesic function that is invariant also w.r.t. mutations of the root edge (at the end of which we set the orbifold point with the parameter $\omega_{0}$ ):

$$
G_{0, i}=b_{i}+c_{i}+\omega_{0} a_{i}
$$

We have thus completely solved the problem of quantum ordering for the product of quantum edge matrices entering the geodesic functions corresponding to geodesics separating exactly two orbifold points from the rest of the Riemann surface. 


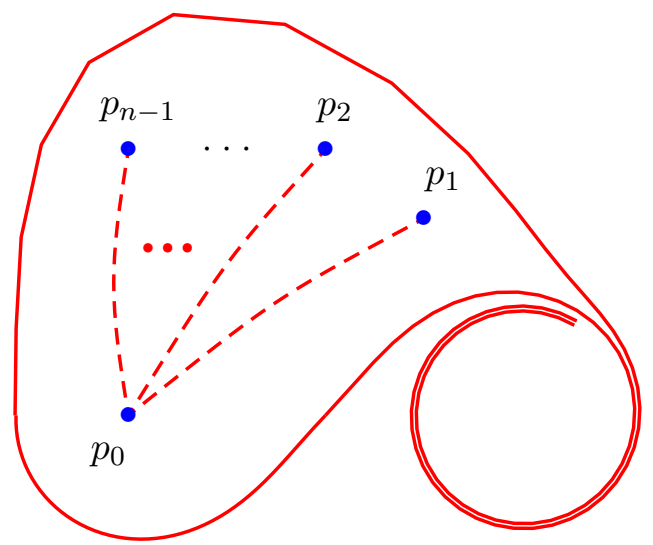

Figure 4: The domain bounded by an infinite geodesic line (a side of a triangle in an ideal triangular decomposition of the Riemann surface) inside which all geodesics $\gamma_{0, i}$ joining orbifold points $p_{0}$ and $p_{i}, i=1, \ldots, n-1$, and constituting the $A_{n}$ system must be contained.

\section{Quantum relations for monodromy matrices}

We know that for some special cases of orbifold Riemann surfaces, the algebra of geodesic functions can be closed. We demonstrate that, in the very same cases, we are also able to close the algebras of elements the Fuchsian group, or monodromy matrices. In this paper, we consider only two such cases: the first one is the $A_{n}$-algebra of a sphere with one hole and with $n \mathbb{Z}_{2}$-orbifold points (in this case, all $\omega_{i}$ are zeros, and $X_{Z} F X_{Z}=X_{2 Z}$ ); the second one is the algebra of geodesic functions on a sphere with four holes/orbifold points of arbitrary orders. We leave to a separate publication the most interesting but also the most difficult case of $D_{n}$-algebras related to reflection equations with the spectral parameter.

\section{1 $A_{n}$-algebra of monodromy matrices}

From the geometry side, we consider a pattern in which $n \mathbb{Z}_{2}$-orbifold points are separated from the rest of the Riemann surface by an infinite geodesic line (a side of an ideal triangle from an ideal triangle decomposition of a finite part of the Riemann surface) whose both ends wind to the perimeter line of the same hole as shown in Fig. 4. The relevant geodesic lines joining the root orbifold point $p_{0}$ with other $n-1$ orbifold points $p_{i}, i=1, \ldots, n-1$, and constituting the $A_{n}$ set must then have no (self)intersections and must be contained inside this (simply connected) domain. We then have a natural linear ordering of these $n-1$ orbifold points ordered counterclockwise.

Given two quantum monodromy matrices $M_{1}$ and $M_{2}$ corresponding to paths joining $p_{0}$ with two other $\mathbb{Z}_{2}$-orbifold points, one of which $\left(p_{1}\right)$ precedes the other $\left(p_{2}\right)$ in the corresponding linear ordering, we can always, using the quantum MCG transformations from the previous sections, reduce them to the case in which (see Fig. 5)

$$
\begin{aligned}
& M_{1}=X_{S} L X_{X} L X_{2 Z} R X_{X} R X_{S}, \\
& M_{2}=X_{S} L X_{X} R X_{2 Y} L X_{X} R X_{S},
\end{aligned}
$$

with the nontrivial quantum commutation relations

$$
\begin{array}{ll}
e^{X} e^{S}=q e^{X+S}, & e^{X} e^{Y}=q e^{X+Y} \\
e^{Y} e^{Z}=q e^{Y+Z}, & e^{Z} e^{X}=q e^{Z+X} .
\end{array}
$$

The both matrices $M_{i}$ have the same structure,

$$
M_{i}=\left[\begin{array}{cc}
q a_{i} & -b_{i} \\
c_{i} & -q^{-1} a_{i}
\end{array}\right]
$$



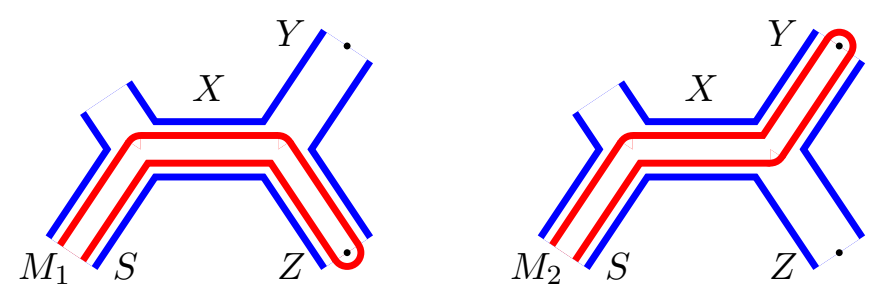

Figure 5: The graphical representation for the monodromy matrices $M_{1}$ and $M_{2}$.

in which we have explicit formulas for $a_{i}, b_{i}$, and $c_{i}$ :

$$
\begin{aligned}
& a_{1}=e^{-X-Z}+e^{-Z} \\
& b_{1}=e^{-X-Z+S}+\left(q+q^{-1}\right) e^{-Z+S}+e^{X-Z+S}+e^{X+Z+S} \\
& c_{1}=e^{-X-Z-S}
\end{aligned}
$$

and

$$
\begin{aligned}
& a_{2}=e^{-X-Y}+e^{-X+Y}+e^{Y} \\
& b_{2}=e^{-X-Y+S}+\left(q+q^{-1}\right) e^{Y+S}+e^{-X+Y+S}+e^{X+Y+S} \\
& c_{2}=e^{-X-Y-S}+e^{-X+Y-S} .
\end{aligned}
$$

In what follows, all $a_{i}, b_{i}$, and $c_{i}$ are Hermitian operators.

Note that the matrices $\tilde{M}_{i}:=F_{0} M_{i}$ corresponding to closed geodesics $\left(F_{0}\right.$ is the matrix with $\left.\omega=0\right)$ satisfy the $U_{q}\left(\mathfrak{s l}_{2}\right)$ relations. We have

$$
\begin{aligned}
& q a_{i} b_{i}-q^{-1} b_{i} a_{i}=0 \\
& q^{-1} a_{i} c_{i}-q c_{i} a_{i}=0, \\
& b_{i} c_{i}-c_{i} b_{i}=\left(q^{2}-q^{-2}\right) a_{i}^{2} \quad \text { in fact } \quad b_{i} c_{i}=q^{2} a_{i}^{2}+1,
\end{aligned} \quad i=1,2
$$

where 1 is the unit element. So, we are in the situation of a quantum algebra with the unity. The above matrices satisfy the quantum relation

$$
M_{i}^{2}=-\mathbb{E}
$$

Interestingly, we can find the complete set of quantum algebraic relations between entries of $M_{1}$ and $M_{2}$, thus leading to a nice algebraic structure on $U_{q}\left(\mathfrak{s l}_{2}\right) \times U_{q}\left(\mathfrak{s l}_{2}\right)$ (we present it in the form closest to the $R$-matrix one):

$$
\begin{aligned}
& q^{-1} b_{1} b_{2}=q b_{2} b_{1}, \\
& q^{-1} c_{1} c_{2}=q c_{2} c_{1}, \\
& a_{1} b_{2}=b_{2} a_{1} \\
& b_{1} a_{2}=a_{2} b_{1}+\left(q^{2}-q^{-2}\right) a_{1} b_{2}=a_{2} b_{1}+\left(q^{2}-q^{-2}\right) b_{2} a_{1}, \\
& c_{1} a_{2}=a_{2} c_{1}, \\
& a_{1} c_{2}=c_{2} a_{1}+\left(q^{2}-q^{-2}\right) c_{1} a_{2}=c_{2} a_{1}+\left(q^{2}-q^{-2}\right) a_{2} c_{1}, \\
& q c_{1} b_{2}=q^{-1} b_{2} c_{1}, \\
& a_{1} a_{2}=a_{2} a_{1}+\left(1-q^{-2}\right) b_{2} c_{1}=a_{2} a_{1}+\left(q^{2}-1\right) c_{1} b_{2}, \\
& q b_{1} c_{2}+q\left[q^{-2}-q^{2}\right] a_{1} a_{2}=q^{-1} c_{2} b_{1}-q^{-1}\left[q^{-2}-q^{2}\right] a_{2} a_{1} .
\end{aligned}
$$


Since we are in a general position case, we can formulate the algebra for any system of $2 \times 2$ matrices $M_{i}, i=1, \ldots, n-1$ such that the paths between the root and all orbifold points are drawn on a tree-like fat subgraph; for simplicity we assume that there exists such a choice of a fat graph in which all these paths are graph-simple, i.e., in representation (31) $Y_{q} \neq Y_{r}$ for $1 \leq q<r \leq p$. We have then the natural linear ordering of the orbifold points from the root of the tree counterclockwise. The algebra of entries $a_{i}, b_{i}, c_{i}$ is given by (38) For $i<j$, we then have the algebra (40) in which we merely substitute $i$ and $j$ for the respective indices 1 and 2 of the elements of monodromy matrices:

$$
\begin{aligned}
& q^{-1} b_{i} b_{j}=q b_{j} b_{i}, \\
& q^{-1} c_{i} c_{j}=q c_{j} c_{i}, \\
& a_{i} b_{j}=b_{j} a_{i}, \\
& b_{i} a_{j}=a_{j} b_{i}+\left(q^{2}-q^{-2}\right) a_{i} b_{j}=a_{j} b_{i}+\left(q^{2}-q^{-2}\right) b_{j} a_{i}, \\
& c_{i} a_{j}=a_{j} c_{i}, \\
& a_{i} c_{j}=c_{j} a_{i}+\left(q^{2}-q^{-2}\right) c_{i} a_{j}=c_{j} a_{i}+\left(q^{2}-q^{-2}\right) a_{j} c_{i}, \\
& q c_{i} b_{j}=q^{-1} b_{j} c_{i}, \\
& a_{i} a_{j}=a_{j} a_{i}+\left(1-q^{-2}\right) b_{j} c_{i}=a_{j} a_{i}+\left(q^{2}-1\right) c_{i} b_{j}, \\
& q b_{i} c_{j}+q\left[q^{-2}-q^{2}\right] a_{i} a_{j}=q^{-1} c_{j} b_{i}-q^{-1}\left[q^{-2}-q^{2}\right] a_{j} a_{i} .
\end{aligned}
$$

Proposition 7. The abstract quantum algebra for $a_{i}, b_{i}, c_{i}, i=1, \ldots, n-1$ defined by the relations (38) and (41) satisfies the quantum Jacobi relation.

Proof. This is a consequence of the fact that the formulae defining $a_{i}, b, c_{i}, i=1, \ldots, n-1$ in terms of the Darboux coordinates are invertible in a large open set.

It is interesting to compare these algebras with the known algebras of traces of products of monodromy matrices like in [6]. Recalling that the monodromy matrix around the root is $M_{0}:=F_{0}=$ $\left[\begin{array}{cc}0 & 1 \\ -1 & 0\end{array}\right]$, the obvious candidates for $G_{0, i}=\operatorname{tr} M_{i} M_{0}$ are linear combinations

$$
G_{0, i}=b_{i}+c_{i} .
$$

A more subtle question is what is $G_{i, j}$, which is the quantum analogue of the trace of product of $M_{i}$ and $M_{j}$ ? Here, we encounter the problem of quantum ordering. From the comparison with the known answer $\left(G_{1,2}=e^{Y+Z}+e^{-Y+Z}+e^{-Y-Z}\right)$ after some algebra we obtain

$$
G_{i, j}=q b_{i} c_{j}+q^{3} c_{i} b_{j}-\left(q^{3}+q\right) a_{i} a_{j}, \quad 1 \leq i<j \leq n-1 .
$$

Let us verify the Hermiticity condition:

$$
\begin{aligned}
G_{1,2}^{*} & =q^{-1} c_{2} b_{1}+q^{-3} b_{2} c_{1}-\left(q^{-3}+q^{-1}\right) a_{2} a_{1} \\
& =q b_{1} c_{2}-\left[q^{2}-q^{-2}\right]\left[q a_{1} a_{2}+q^{-1} a_{2} a_{1}\right]+q^{-1} c_{1} b_{2}-\left(q^{-3}+q^{-1}\right) a_{2} a_{1} \\
& =q b_{1} c_{2}+q^{-1} c_{1} b_{2}-q^{3} a_{1} a_{2}-q a_{2} a_{1}+q^{-1}\left[a_{1}, a_{2}\right] \\
& =q b_{1} c_{2}+q^{-1} c_{1} b_{2}-q^{3} a_{1} a_{2}+q^{-1}\left[q-q^{-1}\right] q c_{1} b_{2}-q a_{2} a_{1} \\
& =q b_{1} c_{2}+q c_{1} b_{2}-q^{3} a_{1} a_{2}-q a_{2} a_{1} \\
& =q b_{1} c_{2}+q c_{1} b_{2}-q^{3} a_{1} a_{2}-q a_{1} a_{2}+q^{2}\left[q-q^{-1}\right] c_{1} b_{2}=G_{1,2} .
\end{aligned}
$$

Using Matematica NCAlgebra package [31] we have verified all the relations of the Nelson-Regge algebra [32, 33], 37]:

$$
\left[G_{i, j}, G_{k, l}\right]=0 \quad \text { for } \quad 0 \leq i<j<k<l \leq n-1 \text { and } 0 \leq i<k<l<j \leq n-1
$$




$$
\begin{aligned}
& {\left[G_{i, k}, G_{j, l}\right]=\left[q^{2}-q^{-2}\right]\left[G_{i, j} G_{k, l}-G_{i, l} G_{j, k}\right] \quad \text { for } \quad 0 \leq i<j<k<l \leq n-1} \\
& q G_{i, j} G_{j, k}-q^{-1} G_{j, k} G_{i, j}=\left[q^{2}-q^{-2}\right] G_{i, k} \quad \text { for } \quad 0 \leq i<j<k \leq n-1, \text { etc. }
\end{aligned}
$$

Remark 5. Note that each monodromy matrix $M_{i}, i=1, \ldots, n-1$, brings two independent quantum operators because we always have one relation (the last formula in (38)) on three additional operators. The same is true for the geometric system: our rooted fat subgraph contains exactly $2 n$ edges, so in this case the geometric system parameterizes the general leaf of the quantum algebra (38), (41).

\section{$5.2 R$-matrix representation for the quantum monodromy $A_{n}$-algebra}

The relations (38), (41) can be presented in the $R$-matrix form. Assuming the standard notation of the tensor product of matrix spaces 1 and 2, we have

$$
\begin{aligned}
& R_{12}[q] \stackrel{1}{M}{ }_{i} R_{12}\left[q^{-1}\right] \stackrel{2}{M}{ }_{j}=\stackrel{2}{M_{j}} R_{12}[q] \stackrel{1}{M} R_{i} R_{12}\left[q^{-1}\right], i<j \\
& R_{12}^{T}\left[q^{-2}\right] \stackrel{2}{M} \stackrel{1}{M}_{i}=\stackrel{2}{M_{i}} \stackrel{M}{M}_{i} R_{12}\left[q^{-2}\right]
\end{aligned}
$$

where

$$
R_{12}[q]:=q\left(E_{11} \otimes E_{11}+E_{22} \otimes E_{22}\right)+E_{11} \otimes E_{22}+E_{22} \otimes E_{11}+\left(q-q^{-1}\right) E_{12} \otimes E_{21}
$$

is the standard $4 \times 4$ quantum $R$-matrix by Kulish and Sklyanin (see [25]),

$$
R_{12}[q]=\left[\begin{array}{cccc}
q & & & \\
& 1 & q-q^{-1} & \\
& & 1 & \\
& & & q
\end{array}\right] .
$$

This matrix possesses the properties

$$
R_{12}\left[q^{-1}\right]=R_{12}^{-1}[q], \quad R_{12}^{T}[q]=R_{21}[q],
$$

where we let the superscript $T$ denote the total transposition.

Note that the reflection equation (44) results in the Jacobi identities for all distinct indices $i, j, k$ provided $R_{12}[q]$ satisfies the quantum Yang-Baxter equation

$$
R_{12}[q] R_{13}[q] R_{23}[q]=R_{23}[q] R_{13}[q] R_{12}[q] .
$$

This follows from the chain of equalities for $i<j<k$ (for brevity, we omit the argument $q$ assuming $\left.R_{12}:=R_{12}[q]\right):$

$$
\begin{aligned}
& {\left[R_{12} \stackrel{1}{M}_{i} R_{12}^{-1} \stackrel{2}{M} j\right] R_{13}^{-1} R_{23}^{-1} \stackrel{3}{M}{ }_{k}=\stackrel{2}{M_{j}} R_{12} \stackrel{1}{M}_{i}\left[R_{12}^{-1} R_{13}^{-1} R_{23}^{-1}\right] \stackrel{3}{M}_{k}} \\
& =\stackrel{2}{M_{j}} R_{12} R_{23}^{-1}\left[\stackrel{1}{M_{i}} R_{13}^{-1} \stackrel{3}{M_{k}}\right] R_{12}^{-1}=\stackrel{2}{M_{j}}\left[R_{12} R_{23}^{-1} R_{13}^{-1}\right] \stackrel{3}{M_{k}} R_{13} \stackrel{1}{M}_{i} R_{13}^{-1} R_{12}^{-1} \\
& =R_{13}^{-1}\left[\stackrel{2}{M}_{j} R_{23}^{-1} \stackrel{3}{M}{ }_{k}\right] R_{12} R_{13} \stackrel{1}{M}_{i} R_{13}^{-1} R_{12}^{-1}=R_{13}^{-1} R_{23}^{-1} \stackrel{3}{M_{k}} R_{23} \stackrel{2}{M}_{j}\left[R_{23}^{-1} R_{12} R_{13}\right] \stackrel{1}{M_{i}} R_{13}^{-1} R_{12}^{-1} \\
& =R_{13}^{-1} R_{23}^{-1} \stackrel{3}{M}{ }_{k} R_{23} R_{13}\left[\stackrel{2}{M}{ }_{j} R_{12} \stackrel{1}{M}_{i}\right]\left[R_{23}^{-1} R_{13}^{-1} R_{12}^{-1}\right] \\
& =R_{13}^{-1} R_{23}^{-1} \stackrel{3}{M}{ }_{k}\left[R_{23} R_{13} R_{12}\right] \stackrel{1}{M_{i}} R_{12}^{-1} \stackrel{2}{M}_{j} R_{12} R_{12}^{-1} R_{13}^{-1} R_{23}^{-1} \\
& =\left[R_{13}^{-1} R_{23}^{-1} R_{12}\right]\left[\stackrel{3}{M}{ }_{k} R_{13} \stackrel{1}{M}_{i}\right] R_{23} R_{12}^{-1} \stackrel{2}{M}_{j} R_{13}^{-1} R_{23}^{-1} \\
& =R_{12} R_{23}^{-1} R_{13}^{-1} R_{13} \stackrel{1}{M}_{i} R_{13}^{-1}\left[\stackrel{3}{M}_{k}\left[R_{13} R_{23} R_{12}^{-1}\right] \stackrel{2}{M_{j}}\right] R_{13}^{-1} R_{23}^{-1} \\
& =R_{12} R_{23}^{-1} \stackrel{1}{M}\left[R_{13}^{-1} R_{12}^{-1} R_{23}\right] \stackrel{2}{M_{j}} R_{23}^{-1} \stackrel{3}{M}=R_{12} \stackrel{1}{M}_{i} R_{12}^{-1} \stackrel{2}{M_{j}} R_{13}^{-1} R_{23}^{-1} \stackrel{3}{M} \text {. }
\end{aligned}
$$


The proof of Jacobi identities for the triple product of entries of the same matrix $M_{i}$ follows from the standard Yang-Baxter equation (48) for the matrices $R_{12}\left[q^{-2}\right]$.

It seems however doubtful that we can write a similar $R$-matrix proof of Jacobi identities in the case where we have two matrices $M_{i}$ and one $M_{j}$ with $i \neq j$. In fact if we did, then we would be able to prove the Jacobi identities for arbitrary matrices $M_{i}$, which is obviously impossible since no such closed algebra exists except a special case of the Painlevé VI algebra considered in Sec. 5.4 below.

\subsection{Braid group action}

As in [6], for $q=1$, we identify the matrices $M_{i}$ with the monodromy matrices of the Fuchsian $2 \times 2$-matrix system:

$$
\partial_{\lambda} \Phi(\lambda)=\sum_{i=0}^{n-1} \frac{A_{i}}{\lambda-u_{i}} \Phi(\lambda) ; \quad \text { eigen } A_{i}=\{1 / 4,-1 / 4\} .
$$

Here $M_{i}$ are monodromy matrices of the 2-vector $\Phi(\lambda)$ when going around the $i$ th singular point $u_{i}$. Choosing $M_{0}=F_{0}$ corresponds to choosing a special basis in the space of solutions: in the vicinity of every singular point $u_{i}$, we have two special solutions $\Phi_{i}^{ \pm}(\lambda)=\left(\lambda-u_{i}\right)^{ \pm 1 / 4} f_{i}^{ \pm}(\lambda)$, with $f_{i}^{ \pm}(\lambda)$ being analytic in the neighborhood of $u_{i}$. Then, obviously, on the classical level our choice of monodromy matrices corresponds to choosing $\Phi^{T}(\lambda)=\left(\Phi_{0}^{+}(\lambda)+\Phi_{0}^{-}(\lambda), \Phi_{0}^{+}(\lambda)-\Phi_{0}^{-}(\lambda)\right)$. The quantum algebra (44), (45) is then the (consistent) quantum analogue of the Korotkin-Samtleben Poisson algebra [24].

The action of the braid group on monodromy matrices was proposed by Dubrovin and M.M. [11]. We have the following lemma in the quantum case.

Lemma 8. The transformations $\beta_{i, i+1}, i=1, \ldots, n-2$, such that

$$
\beta_{i, i+1}\left[M_{j}\right]= \begin{cases}M_{j} & j \neq i, i+1 \\ M_{i} & j=i+1 \\ -M_{i} M_{i+1} M_{i} & j=i\end{cases}
$$

is an automorphism of the quantum algebra (44), (45) and satisfies the quantum braid-group iden-

tities $\beta_{i, i+1} \beta_{i+1, i+2} \beta_{i, i+1}=\beta_{i+1, i+2} \beta_{i, i+1} \beta_{i+1, i+2}$. We again assume the natural ordering of quantum operators in (50).

Proof. The proof of that transformations (50) satisfy the braid group relation repeats the proof of these relations in the classical case (see [11]) provided we have the natural ordering of quantum operators. The proof of that the transformation (50) is an automorphism of the quantum algebra of monodromy matrices is the direct calculation in course of which we have found another nice representation of the braid group action in terms of the quantum geodesic function (43).

The following results can be proved by straightforward computations:

Proposition 9. The quantum determinant relations $b_{i} c_{i}-q^{2} a_{i}^{2}=1, i=1, \ldots, n-1$, are preserved by the quantum braid-group action (50).

Lemma 10. We can present the braid-group action (50) in the form

$$
\beta_{i, i+1}\left[M_{j}\right]= \begin{cases}M_{j} & j \neq i, i+1 \\ M_{i} & j=i+1 \\ q M_{i} G_{i, i+1}-q^{2} M_{i+1}=q^{-1} G_{i, i+1} M_{i}-q^{-2} M_{i+1} & j=i,\end{cases}
$$


and the quantum geodesic operator $G_{i, i+1}$ has the following commutation relations with the monodromy matrices $M_{j}$ :

$$
\begin{aligned}
& q^{-1} G_{i, i+1} M_{i}-q M_{i} G_{i, i+1}=\left(q^{-2}-q^{2}\right) M_{i+1}, \\
& q G_{i, i+1} M_{i+1}-q^{-1} M_{i+1} G_{i, i+1}=\left(q^{2}-q^{-2}\right) M_{i}, \\
& G_{i, i+1} M_{j}-M_{j} G_{i, i+1}=0, \quad j \neq i, i+1 .
\end{aligned}
$$

In general, the quantum geodesic functions $G_{i, j}, 1 \leq i<j \leq n-1$, have the following commutation relations with the quantum monodromy matrices $M_{k}, k=1, \ldots, n-1$ :

$$
\begin{aligned}
& q^{-1} G_{i, j} M_{i}-q M_{i} G_{i, j}=\left(q^{-2}-q^{2}\right) M_{j}, \\
& q G_{i, j} M_{j}-q^{-1} M_{j} G_{i, j}=\left(q^{2}-q^{-2}\right) M_{i}, \\
& G_{i, j} M_{k}-M_{k} G_{i, j}=\left(q^{2}-q^{-2}\right)\left[M_{i} G_{k, j}-G_{i, k} M_{j}\right], \quad i<k<j, \\
& G_{i, j} M_{k}-M_{k} G_{i, j}=0, \quad i<j<k \text { or } k<i<j .
\end{aligned}
$$

Corollary 11. The relations (52) together with the invertibility condition (39) imply that

$$
G_{i, i+1} M_{i} M_{i+1}=M_{i} M_{i+1} G_{i, i+1} \quad \text { and } \quad G_{i, i+1} M_{i+1} M_{i}=M_{i+1} M_{i} G_{i, i+1} .
$$

Therefore the product $M_{1} M_{2} \cdots M_{n-2} M_{n-1}$ and its inverse $M_{n-1} M_{n-2} \cdots M_{2} M_{1}$ are the braid-group invariants.

Remark 6. The fact that we have the braid-group invariants (54) besides the "genuine" Casimirs given by Proposition 9 is due to that we consider here only the subgroup of the total braid group that leaves invariant the rooted edge.

\subsection{Riemann sphere with four holes/orbifold points}

In the case of a Riemann sphere with four holes/orbifold points, the fat graph has a tree-like structure, where the corresponding orbifold points are now of arbitrary orders; we therefore have arbitrary parameters $\omega_{0}, \omega_{1}$ and $\omega_{2}$. Fixing the zeroth hole/orbifold points as root, the arrangement is as in Fig. 6 and we are left with three matrices: $F_{\omega_{0}}$ and another two, more complicated ones, which we call $M_{1}$ and $M_{2}$. The quantum expressions for $M_{1}$ and $M_{2}$ read:

$$
M_{1}=X_{X} L X_{Z} F_{\omega_{1}} X_{Z} R X_{X}=\left[\begin{array}{cc}
q a_{1}+\omega_{1} & -b_{1} \\
c_{1} & -q^{-1} a_{1}
\end{array}\right],
$$

where $a_{1}=e^{-Z}, b_{1}=e^{X-Z}+e^{X+Z}+\omega_{1} e^{X}$, and $c_{1}=e^{-X-Z}$, and

$$
M_{2}=X_{X} R X_{Y} F_{\omega_{2}} X_{Y} L X_{X}=\left[\begin{array}{cc}
q a_{2}+\omega_{2} & -b_{2} \\
c_{2} & -q^{-1} a_{2}
\end{array}\right] \text {, }
$$

where $a_{1}=e^{Y}, b_{1}=e^{X+Y}$, and $c_{1}=e^{-X-Y}+e^{-X+Y}+\omega_{2} e^{-X}$. The entries of both these matrices satisfy the deformed $U_{q}\left(\mathfrak{s l}_{2}\right)$ relations:

$$
\begin{aligned}
& q a_{i} b_{i}=q^{-1} b_{i} a_{i}, \\
& q^{-1} a_{i} c_{i}=q c_{i} a_{i}, \quad i=1,2 \\
& b_{i} c_{i}=1+\omega_{i} q a_{i}+q^{2} a_{i}^{2}, \quad c_{i} b_{i}=1+\omega_{i} q^{-1} a_{i}+q^{-2} a_{i}^{2} .
\end{aligned}
$$



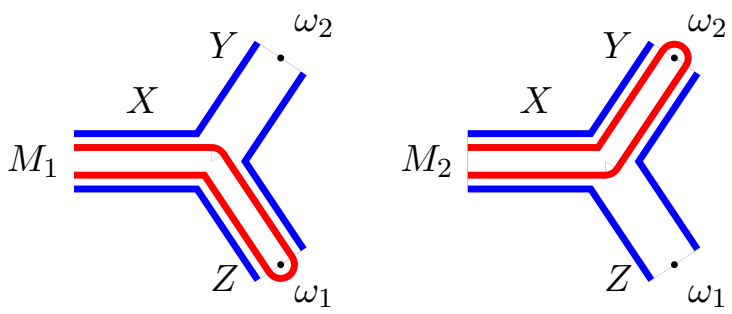

Figure 6: The graphical representation for the monodromy matrices $M_{1}$ and $M_{2}$ in the $\mathrm{P}_{V I}$ case.

The algebraic relations between entries of $M_{1}$ and $M_{2}$ are

$$
\begin{aligned}
& q^{-1} a_{1} a_{2}=q a_{2} a_{1}, \\
& q^{-1} b_{1} b_{2}=q b_{2} b_{1}, \\
& q^{-1} c_{1} c_{2}=q c_{2} c_{1}, \\
& b_{1} a_{2}+q^{-2} a_{1} b_{2}+\omega_{1} q^{-1} b_{2}=a_{2} b_{1}+q^{2} b_{2} a_{1}+\omega_{1} q b_{2}, \\
& a_{1} b_{2}=b_{2} a_{1}, \\
& a_{1} c_{2}+q^{-2} c_{1} a_{2}+\omega_{2} q^{-1} c_{1}=c_{2} a_{1}+q^{2} a_{2} c_{1}+\omega_{2} q c_{1}, \\
& c_{1} a_{2}=a_{2} c_{1}, \\
& q c_{1} b_{2}=q^{-1} b_{2} c_{1}, \\
& q b_{1} c_{2}-q^{-1} c_{2} b_{1}=\left[q^{2}-q^{-2}\right]\left[q a_{1} a_{2}+q^{-1} a_{2} a_{1}+\omega_{1} a_{2}+\omega_{2} a_{1}\right]+\left[q-q^{-1}\right] \omega_{1} \omega_{2} .
\end{aligned}
$$

These relations in general are not consistent (otherwise we were be able to formulate a monodromy algebra of $A_{n}$-type for arbitrary parameters $\omega_{i}$ ). They become consistent if we supply them with an additional condition

$$
a_{1} a_{2}=q^{2} c_{1} b_{2} .
$$

We call the algebraic relations (57)-(59), (60)-(68), and (69) the monodromy algebra for the $\mathrm{P}_{V I}$ equation.

Note that we have three relations, (59) for $i=1,2$ and (69), on six variable $a_{i}, b_{i}, c_{i}$, which implies that our algebra must have an additional central element. It is not difficult to find that the elements

$$
\begin{aligned}
& K_{1}:=a_{1} c_{2}-q^{2} c_{1} a_{2}-q \omega_{2} c_{1} \\
& K_{2}:=a_{2} b_{1}-q^{-2} b_{2} a_{1}-q^{-1} \omega_{1} b_{2}
\end{aligned}
$$

are central and they are related:

$$
K_{1} K_{2}=1
$$

We can thus identify the parameter $\omega_{3}$ corresponding to the fourth hole/orbifold point:

$$
\omega_{3}:=K_{1}+K_{2} .
$$

Remark 7. It is interesting to mention that the monodromy matrices $M_{1}$ and $M_{2}$ given by the respective expressions (55) and (56) satisfy the very same relation (44) with $i=1$ and $j=2$ and with exactly the same $R$-matrix (46) provided the relations (60)-(68) and (69) be satisfied.

We now define the three geodesic functions of the $\mathrm{P}_{V I}$ system:

$$
\begin{aligned}
G_{X Z} & =c_{1}+b_{1}+\omega_{0} a_{1}, \\
G_{X Y} & =c_{2}+b_{2}+\omega_{0} a_{2}, \\
G_{Y Z} & =q b_{1} c_{2}-q^{3} a_{1} a_{2}-q^{2}\left(\omega_{1} a_{2}+\omega_{2} a_{1}\right)-q \omega_{1} \omega_{2},
\end{aligned}
$$


where we have introduced the last missing parameter $\omega_{0}$. For these quantities we then obtain the system of algebraic relations of the $\mathrm{P}_{V I}$ algebra of geodesic functions:

$$
\begin{aligned}
& q G_{X Y} G_{X Z}-q^{-1} G_{X Z} G_{X Y}=\left[q^{2}-q^{-2}\right] G_{Y Z}+\left[q-q^{-1}\right]\left[\omega_{1} \omega_{2}+\omega_{0} \omega_{3}\right], \\
& q G_{X Z} G_{Y Z}-q^{-1} G_{Y Z} G_{X Z}=\left[q^{2}-q^{-2}\right] G_{X Y}+\left[q-q^{-1}\right]\left[\omega_{2} \omega_{0}+\omega_{1} \omega_{3}\right], \\
& q G_{Y Z} G_{X Y}-q^{-1} G_{X Y} G_{Y Z}=\left[q^{2}-q^{-2}\right] G_{X Z}+\left[q-q^{-1}\right]\left[\omega_{0} \omega_{1}+\omega_{2} \omega_{3}\right] .
\end{aligned}
$$

Remark 8. In [26], it was proved that $F_{\omega_{0}}, M_{1}, M_{2}$ and a fourth matrix corresponding to the quantization of their product satisfy the Cherednik algebra $\mathcal{H}$ relations of type $\check{C}_{1} C_{1}$. The algebra (77) corresponds to the spherical subalgebra $e \mathcal{H} e$ of $\mathcal{H}$. In this paper we have shown that $M_{1}$ and $M_{2}$ satisfy the deformed $U_{q}\left(\mathfrak{s l}_{2}\right)$ relations (57), thus providing an interesting relation between the Cherednik algebra of type $\tilde{C}_{1} C_{1}$ and the deformed $U_{q}\left(\mathfrak{s l}_{2}\right)$.

\section{Conclusion}

In conclusion, we recall the main new results obtained in this paper.

We have quantized the MCG transformations (or flip morphisms) for flipping pending edges of graphs corresponding to open Riemann surfaces with orbifold points.

Using the fact that elements of Fuchsian groups transform uniformly under the action of quantum MCG transformations, we were able to provide the explicit quantum ordering for a special class of quantum geodesic functions corresponding to geodesics joining exactly two orbifold points or holes on a non-compact Riemann surface.

We have used the same quantum ordering for quantizing matrix elements of the Fuchsian group associated to the Riemann surface. Interestingly, in all the cases where it is possible to close the Poisson algebra of geodesic functions, such as the $A_{n}$-algebra related to Schlesinger systems [10] and in the case of algebra related to the Painlevé VI equation [7], we have obtained a well defined quantum algebra for the elements in the corresponding Fuchsian group.

In the case of the $A_{n}$-algebra, for each fixed geodesic, the matrix entries of the corresponding element in the Fuchsian group satisfy the quantum universal enveloping algebra $U_{q}\left(\mathfrak{s l}_{2}\right)$ relations, while in the case of algebra related to the Painlevé VI equation they satisfy a deformed version of $U_{q}\left(\mathfrak{s l}_{2}\right)$.

This result is quite interesting as it sheds light on the relation between the quantum universal enveloping algebra $U_{q}\left(\mathfrak{s l}_{2}\right)$ and the Zhedanov algebra $A W(3)$ already explored in [38, 35]. We have also obtained the quantum generalisation of the Korotkin-Samtleben $r$-matrix Poisson algebra for the Schlesinger system and constructed the action of the quantum braid group on the entries of quantum monodromy matrices.

In a separate publication, we shall consider the last remaining case of $D_{n}$-algebras of matrix elements of the Fuchsian group for an annulus with $n \mathbb{Z}_{2}$-orbifold points. The corresponding algebra of quantum geodesic functions is related to the reflection equation with the spectral parameter.

\section{Acknowledgments}

The authors are grateful to P. Wiegmann and A. Zabrodin for useful conversations. This work was supported by the Engineering and Physics Sciences Research Council EP/J007234/1. The work of L.Ch. was also supported in part by the Russian Foundation for Basic Research (Grant Nos. 11-0100440-a and 13-01-12405-ofi-m), by the Grant of Supporting Leading Scientific Schools of the Russian Federation NSh-4612.2012.1, and by the Program Mathematical Methods for Nonlinear Dynamics. 


\section{References}

[1] F. Bonahon, Shearing hyperbolic surfaces, bending pleated surfaces and Thurston's symplectic form, Ann. Fac. Sci. Toulouse Math $6 \mathbf{5}$ (1996), 233-297.

[2] Chekhov L. O., Riemann surfaces with orbifold point, Proc. Steklov Math. Inst., 266 (2009), pp.1-26

[3] Chekhov L. O., Orbifold Riemann surfaces and geodesic algebras, J. Phys. A: Math. Theor. 42 (2009) Paper 304007, 32 pp. (electronic)

[4] Chekhov L., Fock V., A quantum Techmüller space, Theor. and Math. Phys. 120 (1999), 1245-1259, http://arxiv.org/abs/math.QA/9908165math.QA/9908165.

[5] Chekhov L., Fock V., Quantum mapping class group, pentagon relation, and geodesics, Proc. Steklov Math. Inst. 226 (1999), 149-163.

[6] Chekhov L.O., Mazzocco M., Isomonodromic deformations and twisted Yangians arising in Teichmüller theory, Advances Math. 226(6) (2011) 4731-4775, arXiv:0909.5350.

[7] L.O. Chekhov, M. Mazzocco, Shear coordinate description of the quantized versal unfolding of a $D_{4}$ singularity J. Phys. A: Math. Theor. 43 (2010) 442002 (9pp.)

[8] L.O.Chekhov and R.C. Penner, Introduction to quantum Thurston theory, Russ. Math. Surv. 58(6) (2003) 11411183.

[9] L. Chekhov and M. Shapiro Teichmller spaces of Riemann surfaces with orbifold points of arbitrary order and cluster variables, Intl. Math. Res. Notices 2013; doi: 10.1093/imrn/rnt016. (arXiv:1111.3963, 20pp)

[10] Dubrovin B., Geometry of 2D topological field theories, Integrable systems and quantum groups (Montecatini Terme, 1993), Lecture Notes in Math., 1620, Springer, Berlin, (1996) 120-348.

[11] Dubrovin B.A., Mazzocco M., Monodromy of certain Painlevé-VI transcendents and reflection group, Invent. Math. 141 (2000), 55-147.

[12] L. D. Faddeev and R. M. Kashaev, Quantum dilogarithm, Modern Phys. Lett. A9 (1994) 427-434, hep-th/9310070.

[13] L. D. Faddeev, Discrete Heisenberg-Weyl group and modular group, Lett. Math. Phys., 34, (1995), $249-254$.

[14] Fock V.V., Combinatorial description of the moduli space of projective structures, http://arxiv.org/abs/hepth/9312193.

[15] V. V. Fock and A. B. Goncharov, Dual Teichmüller and lamination spaces, Chapter 15 in: Handbook on Teichmuller Theory, Vol.1 (IRMA Lectures in Mathematics and Physics, Vol.11), ed. A.Papadopoulos, IRMA Publ., Strasbourg, France. pp.647-684; math.DG/0510312.

[16] V. V. Fock and A. A. Rosly, Moduli space of flat connections as a Poisson manifold, Internat. J. Modern Phys. B 11 (1997), no. 26-27, 3195-3206.

[17] Fomin S., Shapiro M., Thurston D., Cluster algebras and triangulated surfaces. Part I: Cluster complexes, Acta Math. 201 (2008), no. 1, 83-146.

[18] S. Fomin and A. Zelevinsky, Cluster algebras I: Foundations, J. Amer. Math. Soc. 15(2) (2002) $497-529$.

[19] S. Fomin and A. Zelevinsky, Cluster algebra II: : Finite type classification Invent. Math., 154 (2003), no. 1, 63-121.

[20] Goldman W.M., Invariant functions on Lie groups and Hamiltonian flows of surface group representations, Invent. Math. 85 1986), 263-302.

[21] Ito T. and Terwilliger P., Double affine Hecke algebras of rank 1 and the $\mathbb{Z}_{3}$-symmetric Askey-Wilson relations, SIGMA 6 (2010) 065, 9 pages.

[22] R. M. Kashaev, Quantization of Teichmüller spaces and the quantum dilogarithm, Lett. Math. Phys. 43(1998),105115, q-alg/9706018.

[23] R. M. Kashaev, On the spectrum of Dehn twists in quantum Teichmüller theory, in: Physics and Combinatorics, (Nagoya 2000). River Edge, NJ, World Sci. Publ., 2001, 63-81; math.QA/0008148.

[24] Korotkin D. and Samtleben H., Quantization of coset space $\sigma$-models coupled to two-dimensional gravity, Comm. Math. Phys. 190 (1997), no. 2, 411-457.

[25] P. P. Kulish and E. K. Sklyanin, Quantum spectral transform method: Recent evelopments, in Integrable Quantum Field Theories (Lect. Notes in Physics,: Vol. 151), Berlin, Springer, 1982, pp. 61-119.

[26] Mazzocco M., Confluences of the Painlevé equations, Cherednik algebras and q-Askey scheme, http://arxiv.org/abs/130\%.6140 (2013). 
[27] Molev A., Yangians and classical Lie algebras. Mathematical Surveys and Monographs, 143, American Mathematical Society, Providence, RI, (2007).

[28] A. Molev, E. Ragoucy, Symmetries and invariants of twisted quantum algebras and associated Poisson algebras, Rev. Math. Phys., 20(2) (2008) 173-198.

[29] A. Molev, E. Ragoucy, P. Sorba, Coideal subalgebras in quantum affine algebras, Rev. Math. Phys., 15 (2003) 789-822.

[30] M. L. Nazarov, Quantum Berezinian and the classical Capelli identity, Lett. Math. Phys. 21 (1991) $123-131$.

[31] Hurst D., Lamm D., Merino O., Obar R., Pfister H., Walker M., Wavrik J., Yu L., Camino J., Griffin J., Ovall J., Shaheen T., Shopple J., NCAlgebra, a non-commutative algebra package running under Mathematica, http://www.math.ucsd.edu/ ncalg/.

[32] Nelson J.E., Regge T., Homotopy groups and (2+1)-dimensional quantum gravity, Nucl. Phys. B 328 (1989), 190-199.

[33] Nelson J.E., Regge T., Zertuche F., Homotopy groups and $(2+1)$-dimensional quantum de Sitter gravity, Nucl. Phys. B 339 (1990), 516-532.

[34] Penner R.C., The decorated Teichmüller space of Riemann surfaces, Comm. Math. Phys. 113 (1988), $299-339$.

[35] Terwilliger P., The Universal Askey-Wilson Algebra and the Equitable Presentation of $U_{q}\left(\mathfrak{s l}_{2}\right), S I G M A 7(2011)$, 099, 26 pages.

[36] W. P. Thurston, Minimal stretch maps between hyperbolic surfaces, preprint (1984), math.GT/9801039.

[37] Ugaglia M., On a Poisson structure on the space of Stokes matrices, Int. Math. Res. Not. 1999 (1999), no. 9, 473-493, http://arxiv.org/abs/math.AG/9902045math.AG/9902045.

[38] Wiegmann P. B., Zabrodin A. V., Algebrization of difference eigenvalue equations related to $U_{q}\left(\mathfrak{s l}_{2}\right)$, Nucl. Phys. B 451 (1995) 699-724. 